\title{
Gain scheduling LQI controller design for LPV descriptor systems and motion control of two-link flexible joint robot manipulator
}

\author{
Yusuf Altun* \\ Department of Computer Engineering, Faculty of Engineering, Duzce University, Turkey \\ altunyusf@hotmail.com
}

\section{ARTICLE INFO}

Article history:

Received: 21 November 2017

Accepted: 12 April 2018

Available Online: 27 April 2018

Keywords:

Robotic manipulator

Gain scheduling

$L Q R$ controller

Descriptor system

AMS Classification 2010

70E60, 70Q05, 93B51,93B50, 93C85,

93C40, 93C95, 93D09, 93D21

\begin{abstract}
This paper proposes a gain scheduling linear quadratic integral (LQI) servo controller design, which is derived from linear quadratic regulator (LQR) optimal control, for non-singular linear parameter varying (LPV) descriptor systems. It is assumed that state space matrices are non-singular since many mechanical systems do not have any non-singular matrices such as the natural state space forms of robotic manipulator, pendulum and suspension systems. A controller design is difficult for the systems due to rational LPV case. Therefore, the proposed gain scheduling controller is designed without the difficulty. Accordingly, the motion control design is implemented for two-link flexible joint robotic manipulator. Finally, the control system simulation is performed to prove the applicability and performance.
\end{abstract}

$(c c)$ EY

\section{Introduction}

Many researchers have recently considered the problem of robust controller design on linear parameter varying or linear time invariant systems in the regular statespace form such as in [1-14], where the systems are common linear parameter varying (LPV) systems. Unlike the regular state-space forms, descriptor systems enable an expression which includes algebraic conditions on physical factors. Therefore, these systems have attracted attention for the past decade because many systems such as mechanical systems have this structure, and the systems have ability to describe many systems such as chemical processes, robotic systems, aircrafts, etc. Thus, many researchers have investigated in the literature, for instance, robust stability analysis and stabilisation [15-17], robust controllability/ observability analysis [18], $H_{2} / H_{\infty}$ norm characterization and control [19-27], robust filtering [21], positive real analysis and control [22].

They have two types which are singular and nonsingular descriptor systems. Singular systems, which are also called differential algebraic equations (DAE), are generally used to describe some systems including algebraic constraints. On the other hand, many of mechanical systems such as robotic manipulators, pendulum systems and suspension systems have not any singular matrices although they can be represented as singular system form by including algebraic constraints. This is because, they are naturally in the form of non-singular descriptor state space forms due to using Euler-Lagrange method based on energy for the mathematical modelling. On the other hand, nonsingular LPV descriptor systems can occur rational LPV form due to uncertain parameters, for which the controller design is difficult. Accordingly, the mentioned papers are mostly for the singular systems.

Flexible robotic manipulators have many advantages on the rigid robots which need less material, lighter in weight, less power, smaller actuators, more manoeuvrable and transportable, and thus their cost are lower. These robots have a wide application area as an industrial robot in industry. In addition, two-link flexible manipulators are preferred because they present more flexibility for applications. Nevertheless, their control is difficult to attain exact positioning. Also, the complexity of problem arises because they are multi-input multi-output (MIMO) systems, which are affected by several factors such as payload changing and vibration effects of between links. Moreover, they have nonlinearity and some uncertain parameters. Therefore, numerous researchers have investigated for the control of robotic manipulators in [28-34]. For instance, $H_{\infty}$ control and $\mu$-synthesis are in $[28,29,33]$.

*Corresponding author 
In [30], the control of flexible robotic manipulator is dealt with finite element theory. In [31], a robust control method of a two-link flexible manipulator with neural network. In [32], $H_{\infty}$ control is performed for LPV descriptor model with affine parametric dependence by using a linear fractional representation (LFR). In [34], LPV control is performed by converting equivalent to a rational LPV system. In [35], general $H_{\infty}$ LPV control is designed for the non-singular descriptor flexible robotic manipulator.

Thus, the designs in above papers are mostly for the singular LPV systems. In addition, a gain scheduling Linear Quadratic Integral (LQI) controller has not been tackled for the non-singular descriptor systems in the literature. Accordingly, many mechanical systems are naturally in the form of non-singular LPV system. In this paper, we consider the construction of a gain scheduling LQI controller for a non-singular uncertain descriptor system. The controller is applied to two-link robotic manipulator. Natural two-link robotic manipulator model has not any singular matrices, but it and similar mechanical systems can be converted to singular form by LPV conversion methods as in [22, $36,37]$, which is not in the focus of paper. Because of avoiding the complexity of rational LPV systems, the proposed controller can be applied to a robotic manipulator system or any non-singular LPV descriptor system without using rational LPV form. Finally, the main aim of the paper is to design a static controller without any extra methods and assumptions for the rational LPV systems. From the references and the other literature studies, there is not a gain-scheduling controller LQI design for the LPV descriptor systems without conversion methods.

\section{Two link robotic manipulator model}

The robotic manipulator is shown in Figure 1. It is a two-link flexible planar manipulator which is driven by geared two DC motors. $\theta_{1}$ and $\theta_{2}$ are the shoulder and elbow joint angles, respectively. $\tau_{1}$ and $\tau_{2}$ are the corresponding control torques. The second-order form of the manipulator nonlinear motion equations [28] are as in (1) where $M\left(\theta_{2}\right)$ is the inertia matrix, $D$ is the damping matrix, and $K$ is the stiffness matrix, $F$ is input vector and. $M\left(\theta_{2}\right)$ is as in (2) where $M(\pi)$ and $M(\pi / 2)$ are given by (3).

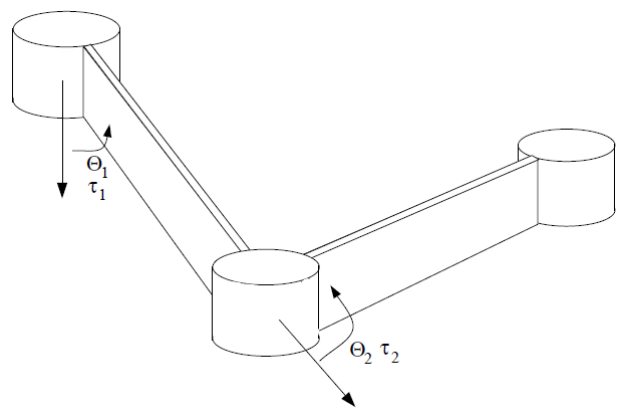

Figure 1. Two link robotic manipulator schema [28].

$$
\begin{aligned}
& M\left(\theta_{2}\right) \ddot{q}(t)+D \dot{q}(t)+K q(t)=F u(t) \\
& M\left(\theta_{2}\right)=M(\pi / 2)+\cos \left(\theta_{2}\right)[M(\pi / 2)-M(\pi)] \\
& \mathrm{M}\left(\frac{\pi}{2}\right)=\left[\begin{array}{cccc}
34.7077 & 9.7246 & 23.6398 & 5.9114 \\
9.7246 & 9.8783 & 9.7246 & 5.9114 \\
23.6398 & 9.7246 & 17.5711 & 5.9114 \\
5.9114 & 5.9114 & 5.9114 & 3.7233
\end{array}\right], \\
& \mathrm{M}(\pi)=\left[\begin{array}{cccc}
17.0296 & 0.8856 & 9.7776 & 0.8430 \\
0.8856 & 9.8783 & 4.7016 & 5.9114 \\
9.7776 & 4.7016 & 7.5249 & 3.0311 \\
0.8430 & 5.9114 & 3.0311 & 3.7233
\end{array}\right]
\end{aligned}
$$

Also, $D$ the damping matrix, and $K$ the stiffness matrix and $F$ input vector are

$$
\begin{aligned}
\mathrm{D} & =\left[\begin{array}{cccc}
0 & 0 & 0 & 0 \\
0 & 0 & 0 & 0 \\
0 & 0 & 0.09 & 0 \\
0 & 0 & 0 & 0.05
\end{array}\right], \\
\mathrm{K} & ==\left[\begin{array}{cccc}
0 & 0 & 0 & 0 \\
0 & 0 & 0 & 0 \\
0 & 0 & 89.1473 & 0 \\
0 & 0 & 0 & 45.6434
\end{array}\right], \mathrm{F}=\left[\begin{array}{ll}
1 & 0 \\
0 & 1 \\
0 & 0 \\
0 & 0
\end{array}\right]
\end{aligned}
$$

\section{Gain-scheduling LQI controller design}

LQR control is an optimal control method, which is commonly used for the state feedback design in the literature. But, it has no output matrix. Therefore, LQI control, which is based on LQR, has the output feedback with integral action. Thus, LQR is regulator while LQI is servo design. That is, LQI controller is used for the reference tracking. In this paper, gain scheduling LQI controller design is proposed for the non-singular LPV descriptor form, and the method is applied to two link robotic manipulator.

$\dot{x}(t)=A x(t)+B u(t), x(0)=x_{0}$

Consider the linear time invariant (LTI) system in (4), $J$ the performance index (or cost functional) is defined in (5) for the state feedback optimal controller $u(t)=-K x(t)$, where $Q^{n \times n}$ is positive semidefinite matrix and $R^{m \times m}$ is positive symmetric matrix. The aim of LQR control is to design a state feedback controller $u(t)=-K x(t)$ which minimizes performance index $J$ and stabilizes the system. In addition, LQI controller is obtained by including output and reference error. Therefore, if integral action is included in system, LQI controller is designed. Also, its effect is to drop steady state errors.

$$
J(t)=\int_{0}^{\infty}\left(x^{T}(t) Q x(t)+u^{T}(t) R u(t)\right) d t
$$


Robotic manipulators are in the form of (6) where timevarying parameter set is given by (7). It is assumed that $E(v)$ is non-singular matrix for all $v$.

$$
\begin{aligned}
& E(v) \dot{x}(t)=A x(t)+B u(t), x(0)=x_{0} \\
& y=C x(t) \\
& v_{D}=\left\{\dot{v}(t) \in \mathfrak{R}^{n}: \overline{\dot{v}_{i}} \leq \dot{v}_{i}(t) \leq \underline{\dot{v}_{i}}, \forall_{i}=1, \ldots, n\right\} \\
& v_{R}=\left\{v(t) \in \mathfrak{R}^{n}: \overline{v_{i}} \leq v_{i}(t) \leq \underline{v_{i}}, \forall_{i}=1, \ldots, n\right\}
\end{aligned}
$$

Their motion control is a reference tracking problem. Therefore, system error is given by.

$$
\begin{aligned}
e(t) & =r(t)-y(t), \\
\dot{e}(t) & =\dot{r}(t)-\dot{y}(t)=\dot{r}(t)-C \dot{x}(t)
\end{aligned}
$$

Accordingly, the extended system is as follows.

$$
\begin{aligned}
& \underbrace{\left[\begin{array}{ll}
E(v) & I
\end{array}\right]}_{\hat{E}}\left[\begin{array}{c}
\ddot{x}(t) \\
\dot{e}(t)
\end{array}\right]=\underbrace{\left[\begin{array}{ll}
A & B \\
C & 0
\end{array}\right]}_{\hat{A}} \underbrace{\left[\begin{array}{c}
\dot{x}(t) \\
e(t)
\end{array}\right]}_{\hat{x}}+\left[\begin{array}{c}
B \\
0
\end{array}\right] \dot{u}(t), \\
& \hat{x}(0)=\hat{x}_{0}
\end{aligned}
$$

So, LQI controller including output feedback is as follows.

$$
K_{L Q I}(v(t)) \hat{x}(t)=K_{1}(v(t)) x(t)+K_{2}(v(t)) \int_{0}^{t} y(t) .
$$

Accordingly, the following theorem presents the proposed gain scheduling LQI controller design.

Theorem: Consider a non-singular descriptor LPV system in (6) and (8), an optimal controller input $K_{L Q I}(v(t)) \hat{x}(t)=K_{1}(v(t)) x(t)+K_{2}(v(t)) \int_{0}^{t} y(t) \quad$ and controller matrix in (10) which minimizes the performance index $J$ in (5), if and only if there exist parameter-dependent symmetric positive-definite matrices $Y \in \mathfrak{R}^{n \times n}, Z(v) \in \mathfrak{R}^{q \times q}$ and block diagonal positive-definite matrix $X \in \mathfrak{R}^{n \times n}$, the inequalities in (9) hold all for all $v(t) \in v_{R} \times v_{D}$.

$\min \gamma$, such that

$$
\begin{aligned}
& {\left[\begin{array}{cc}
\hat{A} Y \hat{E}^{T}(v)+(*)^{T}-\hat{B} Z(v) \hat{B}^{T} & \hat{E}(v) Y \\
Y \hat{E}^{T}(v) & X
\end{array}\right]<0} \\
& {\left[\begin{array}{cc}
-\gamma & \hat{x}_{0}^{T} \\
\hat{x}_{0} & -Y
\end{array}\right]<0} \\
& K_{L Q I}(v)=Z(v) \hat{B}^{T} Y^{-1}
\end{aligned}
$$

Proof: The non-singular descriptor system in (8) is rewritten as follows.

$$
\dot{\hat{x}}(t)=\hat{E}(v)^{-1} \hat{A} \hat{x}(t)+\hat{E}(v)^{-1} \hat{B} \hat{u}(t), \hat{x}(0)=\hat{x}_{0}
$$

When Lyapunov stability criteria is applied for asymptotic stability for Lyapunov function $V=\hat{x}^{T} P \hat{x}$, the following condition should be provided [38]:

If the controller $\hat{u}(t)=-K_{L Q I}(v) \hat{x}(t)$ provides the condition $\frac{d V}{d t} \leq 0$, the system is stable and (12) is obtained for the performance index. If $V=\hat{x}^{T} P \hat{x}$ is replaced, the optimal controller in (13) is obtained.

$\frac{d V}{d t}+\hat{x}^{T}(t) Q \hat{x}(t)+\hat{u}^{T}(t) R(v) \hat{u}(t)=0$

$\hat{u}(t)=-R^{-1}(v) \hat{B}^{T} P \hat{x}(t)=-K(v) \hat{x}(t)$

In that case, algebraic Riccati matrix equation in (14) is yielded.

$\hat{A}^{T} \hat{E}^{-T}(v) P+P \hat{E}^{-1}(v) \hat{A}$

$-P \hat{E}^{-1}(v) \hat{B} R^{-1}(v) \hat{B}^{T} \hat{E}^{-T}(v) P+Q=0$

The matrix equation is converted to Linear matrix inequality (LMI) because LMI approach is proposed for the design. Because, it is commonly used for the controller design since LMI approach has many advantages.

For minimizing the cost function, the following equality is obtained. The matrix equation in (14) together with initial condition can be expressed

$$
\begin{aligned}
& J_{\min }(t)=\min \int_{0}^{\infty}\left[\hat{x}^{T}(t) Q \hat{x}(t)+\hat{u}^{T}(t) R(v) \hat{u}(t)\right] d t \\
& =\hat{x}_{0}^{T}(t) P \hat{x}_{0}(t)
\end{aligned}
$$

Accordingly, following Schur formula in [38] is commonly used in linear algebra applications:

Consider a symmetric matrix $T=\left[\begin{array}{cc}T_{11} & T_{12} \\ T_{12}^{T} & T_{22}\end{array}\right], T<0$, if and only if $T_{22}<0$ and $T_{11}-T_{12} T_{22}^{-1} T_{12}^{T}<0$.

Thus, if (14) is multiplied with $P^{-1}=Y$, LMI form in (16) can be written as follows. And then, if Schur formula is applied, the inequality in (17) is obtained.

$$
\begin{aligned}
& \hat{E}^{-1}(v) \hat{A} Y+Y \hat{A}^{T} \hat{E}^{-T}(v) \\
& -\hat{E}^{-1}(v) \hat{B} R^{-1}(v) \hat{B}^{T} \hat{E}^{-T}(v)+Y Q Y<0 \\
& {\left[\begin{array}{cc}
\left(\hat{E}^{-1}(v) \hat{A} Y+(*)^{T}\right. & Y \\
-\hat{E}^{-1}(v) \hat{B} R^{-1}(v) \hat{B}^{T} \hat{E}^{-T}(v) & \\
Y & Q^{-1}
\end{array}\right]<0}
\end{aligned}
$$

The matrix $P\left(P^{-1}=Y\right)$ is minimized to get minimum performance index. Therefore, defining $\gamma, \gamma>\hat{x}_{0}^{T} P \hat{x}_{0}$ can be obtained. Then, if Schur formula is applied to $\hat{x}_{0}^{T} P \hat{x}_{0}-\gamma<0$, the inequality in (18) is obtained. 




Figure 2. Control system diagram.

$$
\left[\begin{array}{cc}
-\gamma & \hat{x}_{0}^{T} \\
\hat{x}_{0} & -Y
\end{array}\right]<0
$$

The inequality in (17) is multiplied from left and right with the matrix in (19) and its transpose, respectively.

$$
\left[\begin{array}{cc}
\hat{E}(v) & 0 \\
0 & I
\end{array}\right]
$$

Thus, we get the following inequality by also defining $X=Q^{-1}$ and $Z(v)=R^{-1}(v)$, and the proof completes.

$$
\left[\begin{array}{cc}
\hat{A} Y \hat{E}^{T}(v)+(*)^{T}-\hat{B} Z(v) \hat{B}^{T} & \hat{E}(v) Y \\
Y \hat{E}^{T}(v) & X
\end{array}\right]<0
$$

Finally, an optimal feedback controller $K_{L Q I}(v(t)) \hat{x}(t)=K_{1}(v(t)) x(t)+K_{2}(v(t)) \int_{0}^{t} y(t)$,

which minimizes performance index $J$ and stabilizes the system, can be obtained by solving the optimization problem in (9) for the system in (8) including output feedback.

Remark: The inverse of $E(v)$ is a drastic case due to rational LPV form and there is no a formula for this, so an extra complexity occurs, and some assumptions or extra methods are needed as in [22, 28, 36, 37]. For example, since $\frac{A(\theta)}{E(\theta)}=E^{-1}(\theta) A(\theta)$ is dependent a parameter theta, the making inverse of the parameter dependent matrix is a difficult problem. But, the proposed solution eliminates to take its inverse. Thus, the proposed design has no the complexity and does not need any extra methods.

\section{Simulation results}

The simulation tests are performed with MATLAB. Figure 2 shows the simulation block diagram for the two-link flexible manipulator control system. The timevarying parameter is assumed as follows for robotic manipulator system.

$$
v(t)=\cos \left(\theta_{2}\right)
$$

Therefore, the bounds of the time-varying parameter is $-1 \leq v(t) \leq 1$.

Throughout the paper, affine parametric uncertainties are defined for the controller design, and so all parameter-dependent matrices affinely depend on the uncertain parameter such as the controller matrix in (21).

$$
K_{L Q I}(v)=K_{0}+\sum_{i=1}^{n} v_{i} K_{i}
$$

In addition, the selected matrix $X$ is for the state weights in the optimization problem in (9). The determination of this matrix is not dependent on the certain rule, but it is optimized by some artificial intelligence methods in the literature. In these paper, classical approach (trial and error) is used according to system behaviour.

$$
X=\operatorname{blkdiag}\left(10^{6} \times(2050,200,16,9,7,1.2,2.3,3.0,0.05,0.03)\right)
$$

If the optimization problem is solved, the effects on the system of controller in (21) are presented in Figure 3, Figure 4 and Figure 5. Figure 3 shows joint angles $\theta_{1}$ and $\theta_{2}$ of robotic manipulator as degree. Thus, the tracking performance is good. The settling times are nearly 3 and 4 second for $\theta_{1}$ and $c$, respectively. Figure 4 shows the time-varying parameter $v(t)$, and accordingly Figure 5 shows the generated torques by the parameter dependent controller as $\mathrm{Nm}$. the results show that the proposed method has good performance such as low settling time and overshoot. The proposed controller is static feedback which changes with $\theta_{2}$, and, there is no assumptions while the controller in [29] is dynamic controller, and there are some asumptions such as fixed angles. Nevertheless, the results for the proposed design are somewhat better than the results in [29] especially for $\theta_{2}$ in point of settling time.

\section{Conclusions}

In this paper, we have addressed the construction of parameter-dependent LQI controller design for the nonsingular LPV descriptor systems, and it has been applied to two-link robotic manipulator. The results have shown that good tracking performances are 
obtained for the joint angle references in view of the settling time and overshoot. In addition, the proposed method has not needed any extra requirements such as rational LPV conversion methods. Finally, the gain scheduling LQI controller has been designed for the non-singular descriptor system without any extra methods for the rational LPV form.
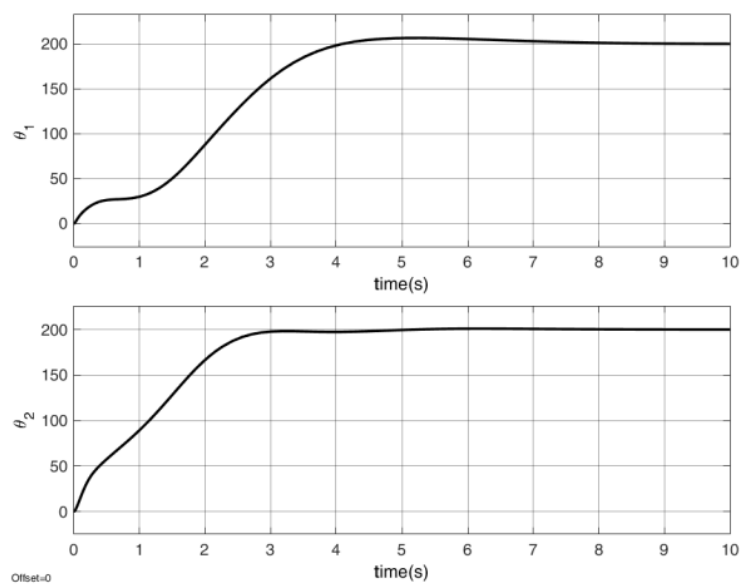

Figure 3. Tracking responses of joint angles.



Figure 4. The change of time-varying parameter $v(t)$.

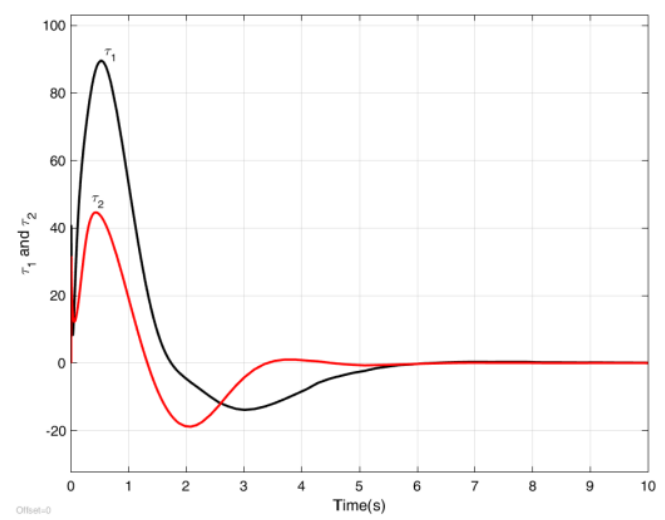

Figure 5. Torque responses.

\section{References}

[1] Park BY, Yun SW, Park P (2012) H 2 statefeedback control for LPV systems with input saturation and matched disturbance. Nonlinear Dynamics 67(2):1083-1096.

[2] Venkataraman R, Seiler P (2018) Convex LPV synthesis of estimators and feedforwards using duality and integral quadratic constraints. International Journal of Robust and Nonlinear Control 28(3):953-975.

[3] He Z, Xie W (2014) Quadratic H2 gain performance analysis of LPV systems with realization of parametric transfer function. Proceedings of the 33rd Chinese Control Conference, CCC 2014 (IEEE), pp 1788-1792.

[4] Peng C, Zhang Z, Zou J, Li K, Zhang J (2013) Internal model based robust inversion feedforward and feedback 2DOF control for LPV system with disturbance. Journal of Process Control 23(10):1415-1425.

[5] Ku C-C, Chen G-W (2015) Gain-scheduled controller design for discrete-time Linear Parameter Varying systems with multiplicative noises. International Journal of Control, Automation and Systems 13(6):1382-1390.

[6] Wu K, Zhang Q, Hansen A (2004) Modelling and identification of a hydrostatic transmission hardware-in-the-loop simulator. Int J Veh Des 34(1):63-75.

[7] Lu B, Choi H, Buckner GD, Tammi K (2008) Linear parameter-varying techniques for control of a magnetic bearing system. Control Engineering Practice 16(10):1161-1172.

[8] Altun Y (2017) A new extended LMI-based robust gain scheduled state feedback $\mathrm{H} 2$ controller design. International Journal of Control, Automation and Systems 15(3):967-975.

[9] Masubuchi I, Kurata I (2011) Gain-scheduled control via filtered scheduling parameters. Automatica 47(8):1821-1826.

[10] Abdullah AA (2018) Robust model reference control of linear parameter-varying systems with disturbances. IET Control Theory \& Applications 12(1):45-52.

[11] Apkarian P, Adams RJ (1998) Advanced gainscheduling techniques for uncertain systems. IEEE Transactions on Control Systems Technology 6(1):21-32.

[12] Huang Y, Na J, Wu X, Gao G-B, Guo Y (2016) Robust adaptive control for vehicle active suspension systems with uncertain dynamics. Transactions of the Institute of Measurement and Control 40(4):1237-1249.

[13] Altun Y (2017) The road disturbance attenuation for quarter car active suspension system via a new static two-degree-of-freedom design. An International Journal of Optimization and Control: Theories \& Applications (IJOCTA) 7(2):142-148.

[14] Yazici H, Sever M, Sever M (2017) Design of an optimal state derivative feedback LQR controller and its application to an offshore steel jacket platform. An International Journal of Optimization and Control: Theories \& Applications (IJOCTA) 8(1):84-91. 
[15] Lin C, Wang QG, Lee TH (2005) Robust normalization and stabilization of uncertain descriptor systems with norm-bounded perturbations. IEEE Transactions on Automatic Control 50(4):515-520.

[16] Fang C-HFC-H (2002) Stability robustness analysis of uncertain descriptor systems - an LMI approach. Proceedings of the 41st IEEE Conference on Decision and Control, 2002 2(December):1459-1460.

[17] Lin C, Wang J, Wang D, Soh CB (1997) Robustness of uncertain descriptor systems. Systems \& Control Letters 31(3):129-138.

[18] Chou JH, Chen SH, Zhang QL (2006) Robust controllability for linear uncertain descriptor systems. Linear Algebra and Its Applications 414(2-3):632-651.

[19] Osorio-Gordillo GL, Darouach M, AstorgaZaragoza CM (2015) Hळ dynamical observers design for linear descriptor systems. Application to state and unknown input estimation. European Journal of Control 26:35-43.

[20] Bara GI (2011) Dilated LMI conditions for timevarying polytopic descriptor systems: The discrete-time case. International Journal of Control 84(6):1010-1023.

[21] Masubuchi I, Suzuki A (2008) Gain-Scheduled Controller Synthesis Based on New LMIs for Dissipativity of Descriptor LPV Systems. IFAC Proceedings Volumes 41(2):9993-9998.

[22] Bouali A, Yagoubi M, Chevrel P (2008) H2 Hळ gain scheduling control for rational LPV systems using the descriptor framework. IEEE Conference on Decision and Control (CDC) (IEEE), pp 38783883.

[23] Rodrigues M, Hamdi H, Benhadj Braiek N, Theilliol D (2014) Observer-based fault tolerant control design for a class of LPV descriptor systems. Journal of the Franklin Institute 351(6):3104-3125.

[24] Ali HS, Darouach M, Zasadzinski M, Alma M (2015) An Ho LPV control for a class of LPV systems using a descriptor approach: Application to a wind turbine mode. IFAC-PapersOnLine 48(26):213-217.

[25] Bara GI (2011) Robust analysis and control of parameter-dependent uncertain descriptor systems. Systems and Control Letters 60(5):356364.

[26] Altun Y (2017) Control Design for Nonsingular Descriptor Systems. Karaelmas Science and Engineering Journal 7(1):154-159.

[27] Bouali A, Yagoubi M, Chevrel P (2008) Gain scheduled observer state feedback controller for rational LPV systems. IFAC Proceedings
Volumes 41(2):4922-4927.

[28] Adams R, Apkarian P, Chretien J (1996) Robust Control Approaches for a Two-Link Flexible Manipulator. 3rd International Conference on Dynamics and Control of Structures in Space, $\mathrm{p}$ 101-116.

[29] Karkoub M, Tamma K, Balas G (1999) Robust Control of Two-Link Flexible Manipulators Using the -Synthesis Technique. Journal of Vibration and Control 5(4):559-576.

[30] Karagülle H, Malgaca L, Dirilmiş M, Akdağ M, Yavuz Ş (2015) Vibration control of a two-link flexible manipulator. Journal of Vibration and Control 23(12):2023-2034.

[31] Li Y, Liu G, Hong T, Liu K (2005) Robust control of a two-link flexible manipulator with quasistatic deflection compensation using neural networks. Journal of Intelligent and Robotic Systems: Theory and Applications 44(3):263-276.

[32] Halalchi H, Laroche E, Bara GI (2010) LPV Modeling and Control of a 2-DOF Robotic. 11th Pan-American Congress of Applied Mechanics.

[33] Yadav PS, Singh N (2015) Robust Control of Two Link Rigid Manipulator. International Journal of Information and Electronics Engineering 5(3):38.

[34] Halalchi H, Laroche E, Iuliana Bara G (2014) Flexible-Link Robot Control Using a Linear Parameter Varying Systems Methodology. International Journal of Advanced Robotic Systems 11(3):46.

[35] Polat I, Eskinat E, Kose IE (2007) Dynamic output feedback control of quasi-LPV mechanical systems. IET Control Theory and Applications 1(4):1114-1121.

[36] Bouali A, Yagoubi M, Chevrel P (2008) H2 gain scheduled observer based controllers for rational LPV systems. 2008 10th International Conference on Control, Automation, Robotics and Vision, ICARCV 2008 41(2):1811-1816.

[37] Galvão RKH, Kienitz KH, Hadjiloucas S (2017) Conversion of descriptor representations to statespace form: an extension of the shuffle algorithm. International Journal of Control:1-15.

[38] Boyd S, El Ghaoui L, Feron E, Balakrishnan V (1994) Linear matrix inequalities in system and control theory, Society for Industrial and Applied Mathematics, Philadelphia.

Yusuf Altun received the Doctoral degree in Electrical Engineering from Yildiz Technical University, Istanbul, Turkey in 2012. He has worked as Assistant Professor at the Duzce University, in Department of Computer Engineering, Duzce, in Turkey since 2013. His main research areas are the control of mechatronic systems such as vehicles, robot, and electromechanical systems. 
An International Journal of Optimization and Control: Theories \& Applications (http://ijocta.balikesir.edu.tr)



This work is licensed under a Creative Commons Attribution 4.0 International License. The authors retain ownership of the copyright for their article, but they allow anyone to download, reuse, reprint, modify, distribute, and/or copy articles in IJOCTA, so long as the original authors and source are credited. To see the complete license contents, please visit http://creativecommons.org/licenses/by/4.0/. 